\title{
Sleep-related Cognitions Mediate the Impact of Neuroticism on Insomnia
}

\author{
Bruno Chauvin, PhD; Anne Thibault-Stoll, MD; Serge Chassagnon, MD, PhD; \\ Sylvie Biry, MPH; Christophe Petiau, MD; Patricia Tassi, PhD
}

Objectives: Our study aimed to explore how neuroticism and neuroticism-related traits as well as sleep-related cognitions (dysfunctional beliefs and subjective qual- ity of sleep) influence the emergence of insomnia using a mediational model. Meth- ods: A cross-sectional study was conduct- ed in which 159 insomniac patients paired with 159 normal sleepers in sex and age $(\mathrm{N}$

$=318$ ) completed an online questionnaire. Results: At the global level, dysfunctional beliefs and poor subjective quality of sleep mediated the neuroticism-insomnia path; at the trait-specific level, these variables mediated the anxiety-insomnia path and partially mediated the effects of vulnerability and self-consciousness on insom- nia; some other relations were essentially indirect effects (between depression and insomnia). Conclusions: These findings extend our understanding of how neuroti- cism is a predisposing factor of insomnia. This knowledge could be helpful to shape prevention and intervention programs to treat insomnia.

Key words: primary insomnia; neuroticism; sleep-related cognitions; path analysis; mediational model
$\mathrm{P}$ rimary insomnia is a prevalent sleep disorder that affects $15 \%$ of the general population. It is defined as difficulty in initiating or maintaining sleep, waking up too early, or sleep that is chronically non-restorative or of poor quality. ${ }^{1}$ Complex interactions among predisposing, precipitating, and perpetuating factors are thought to maintain insomnia over time.

Predisposing factors include personality. Neuroticism and neuroticism related-traits in particular have been identified as key contributors as insomniacs display significantly higher levels of neuroticism than normal sleepers..$^{2-5}$ In a review including 38 studies focused on personality and insomnia, Van de Laar et $\mathrm{al}^{5}$ reported that insomniacs typically showed signs of high neuroticism and traits associated with perfectionism. They tend to exhibit more anxiety in different areas and seem to be more introverted, expressing less easily their negative feelings ("internalization" hypothesis). Other studies show that anxiety and depressive symptoms appear to be important psychological risk factors as-

Bruno Chauvin, University of Strasbourg, Department of Psychology, Strasbourg, France. Anne Thibault-Stoll, Serge Chassagnon, Sylvie Biry, and Christophe Petiau, Clinique Ste Barbe, Groupe Hospitalier St Vincent, Strasbourg, France. Patricia Tassi, University of Strasbourg, Department of Psychology, Strasbourg, France.

Correspondence Dr Chauvin; bruno.chauvin@unistra.fr sociated with the onset of insomnia in the general population, ${ }^{6,7}$ and that higher scores of anger and lower scores of control are associated with longer sleep-onset latency. ${ }^{8}$ Research comparing insomniacs with normal sleepers report that insomniacs score higher on neuroticism-anxiety and impulsivity than non-insomniacs. ${ }^{9}$ Finally, a study by Morin et $a 1,{ }^{10}$ also comparing insomniacs with normal sleepers, suggests that the appraisal of stressors and the perceived lack of control over stressful events can enhance the vulnerability to insomnia.

Perpetuating factors include factors such as sleep-related cognitions because dysfunctional beliefs about sleep (eg, worry over sleep loss) are positively related to insomnia severity, ${ }^{11,12}$ and distorted sleep perceptions (ie, tendency toward overestimating sleep onset latency and underestimating total sleep time) contribute to the maintenance of insomnia. ${ }^{13}$

Taken together, these findings provide a large set of predictors of insomnia. However, the specific role of these factors in the etiology of insomnia remains unclear. It is unknown whether or not neuroticism contributes to insomnia directly or through sleep-related cognitions. Another issue is whether or not the mechanism of the relationship between neuroticism and insomnia is different depending on neuroticism-related traits. These issues are of importance in the context of therapeutic strategies in the sense that cognitive behavioral therapy for 
insomnia (CBT-I) could be adapted to focus on the most relevant dysfunctional cognitions.

The current study directly addressed these issues. We explore the associations among neuroticism (and then neuroticism-related traits), sleeprelated cognitions, and insomnia by means of mediational models and propose structured path models of these psychological factors and their impact on insomnia. Based on studies that found neuroticism to be the first predictor of sleep disturbance before dysfunctional beliefs, ${ }^{14}$ and that neuroticism was associated with insomnia severity, notably via its relationship with problematic sleep-related cognitions, ${ }^{15}$ we propose that neuroticism and neuroticism-related traits are the main predictors of insomnia, while sleep-related cognitions work as mediators of these relations (see also Fernandez-Mendoza et $\mathrm{al}^{16}$ ).

Accordingly, we expect that high neuroticism as well as high scores on neuroticism-related traits should be positively associated with insomnia, more dysfunctional beliefs, and lower levels of subjective sleep quality; we also expect that dysfunctional beliefs and subjective sleep quality should be positively related to insomnia. Finally, we expect that dysfunctional beliefs and subjective sleep quality should mediate the effects of neuroticism and neuroticism-related traits on insomnia.

\section{METHODS}

Participants and Procedure

Two-hundred referred insomniacs who currently receive cognitive behavioral therapy for insomnia (CBT-I) as out-patients in a sleep clinic were contacted through email and accepted to take part in this study. They were diagnosed by sleep specialists as insomniacs because they met the general criterion recommended by the DSM-IV-R. ${ }^{1}$ All of these participants mentioned at least one of the following complaints occurring at least 3 times/week and at least during one month - difficulties in getting to sleep (> $30 \mathrm{~min}$ ), difficulties in maintaining sleep (waking up $>3$ times/night and taking $>30 \mathrm{~min}$ to go back to sleep), experiencing non-restorative sleep (ie, feeling unfreshed in the morning), or early awakening (> $60 \mathrm{~min}$ before normal wake time). Of these, 41 (21\%) were excluded because they also reported the concurrent presence of a sleep disorder other than insomnia (sleep apnea, hypersomnia, and/or restless legs), a serious medical illness like epilepsy, diabetes, multiple sclerosis, and/or a psychiatric disorder such as major depressive or anxiety disorder (ie, all the pathologies likely to affect the sleep quality and quantity for reasons other than primary insomnia). However, for patients in this group who did not report any serious psychiatric disorder, a certain amount of depression and/or anxiety cannot be ruled out altogether, inasmuch as insomniacs often suffer depression and anxious feelings consecutively at chronic sleep debt. Intake of sleep medication was allowed, because almost all the insomniacs used hypnotics (BZD, Zolpidem or Zopiclone), but excessive consumers (> 2 sleeping pills $/ 24$ hours) were excluded. As a result, the sample of insomniacs included a total of 159 participants. This sample consisted of 44 men and 115 women, with a mean age of 49.6 years $(S D=13.7)$. This sex ratio reflects the natural prevalence of insomnia in the general population.

The control group was sampled through staff councils of the University of Strasbourg who were contacted by email and asked to send the Internet link on a voluntary basis to their own mailing lists. Four-hundred participants answered the online questionnaire. Data from 166 participants (42\%) were removed due to not completing the entire questionnaire, resulting in a total of 234 participants who fully completed the questionnaire. All of them were free of insomnia and the other sleep, medical, or psychiatric disorders described above, and did not consume any psychotropic drug. Special attention was given to sex and age in the matching process between insomniacs and normal sleepers because of the relationship between these demographic variables and insomnia. Therefore, of the 234 participants, 35 women aged from 20 to 40 years, and 40 men aged from 20 to 70 years, were randomly selected and excluded, resulting in a sample of 159 normal sleepers including 44 men and 115 women, with a mean age of 48.7 years $(\mathrm{SD}=13)$. Accordingly, 318 participants (159 insomniacs and 159 normal sleepers) were used in the analyses.

\section{Material}

The online questionnaire was divided into $3 \mathrm{sec}-$ tions completed by all participants. In the first section, demographic data (sex, age, level of education, and employment) were collected using open-ended questions. After the matching process, there was no difference of sex or age between insomniacs and normal sleepers. In addition, there was no across-group difference on level of education and employment. The 2 other sections concerned sleep measures (and health-related disorders) and personality.

Sleep measures. Insomnia was assessed through a Yes / No question including all criteria of insomnia as defined by the DSM-IV-R. ${ }^{1}$ The question was: "Do you have difficulties to get asleep, to maintain sleep, early awakening or non-restorative sleep at least 3 times a week for at least one month?" All insomniacs answered the question "Yes" and all normal sleepers answered "No." This measure of insomnia was used as a dichotomous dependent variable (DV). Sleep disorders other than insomnia (sleep apnea, hypersomnia, and restless legs) as well as other health-related problems (serious medical illnesses, psychiatric disorders, use of sleep medication) also were evaluated by Yes/No questions. In addition, all participants were asked to complete a series of items taken from 2 validated self-report measures of sleep disturbances (the Dysfunctional Beliefs and Attitudes about Sleep Scale, DBAS ${ }^{17}$ and the Pittsburgh Sleep Quality Index, PSQI ${ }^{18}$ ). 


\begin{tabular}{|c|c|c|c|c|}
\hline \multicolumn{5}{|c|}{$\begin{array}{c}\text { Table } 1 \\
\text { Correlations between Neuroticism (Factor and Traits) and } \\
\text { Sleep-Related Variables }\end{array}$} \\
\hline \multirow[b]{2}{*}{ Neuroticism } & & \multicolumn{3}{|c|}{ Sleep-related Variables } \\
\hline & & Insomnia & Dysfunctional Beliefs & Subjective Sleep Quality \\
\hline Factor & & .34 & .46 & .38 \\
\hline \multirow[t]{6}{*}{ Traits } & Anxiety & .37 & .46 & .42 \\
\hline & Angry-Hostility & .31 & .32 & .27 \\
\hline & Depression & .34 & .47 & .35 \\
\hline & Self-Consciousness & .16 & .38 & .21 \\
\hline & Impulsiveness & .17 & .18 & $.13^{*}$ \\
\hline & Vulnerability & .41 & .46 & .37 \\
\hline
\end{tabular}

This set of items was selected to assess 2 types of sleep-related cognitions - dysfunctional beliefs about sleep (eg, "I worry about a possible loss of control over the ability to sleep") and perception of sleep quality (eg, "Overall, I rate my sleep quality as good"). For these items, responses were given on a 5-point scale ranged from "strongly disagree" to "strongly agree." Scores for dysfunctional beliefs and subjective sleep quality were created by averaging participants' scores on the corresponding items. Higher scores indicated more dysfunctional beliefs and a poorer subjective quality of sleep. Alpha values were .80 for dysfunctional beliefs and .75 for the subjective quality of sleep.

Personality. Personality was measured through the revised version of the Neuroticism Extraversion Openness Personality Inventory. ${ }^{19}$ This is a 240item questionnaire measuring 5 personality factors called Neuroticism, Extraversion, Conscientiousness, Openness, and Agreeableness (48 items per factor). Each of these factors is an aggregation of 6 narrower aspects called traits of personality (8 items per trait). In this study, based on previous empirical findings about the relationship between insomnia and neuroticism as well as neuroticismrelated traits, we focused on the neuroticism factor and its 6 affiliated traits: Anxiety, Depression, Angry-hostility, Self-consciousness, Impulsiveness, and Vulnerability. Respondents scored each of the 48 items using a 5-point scale varying from "strongly disagree" to "strongly agree." For the neuroticism factor, a mean score was computed by averaging the scores of a 12-item subset (which is approximately comparable to the number of items included in each trait, viz. 8) designed by McCrae and Costa $^{20}$ to represent a short version of the neuroticism factor. For each trait of neuroticism, a mean score was computed by averaging the scores of the 8 corresponding items. Alpha values were .79 for neuroticism, .86 for anxiety, .76 for angry-hostility, .83 for depression, .70 for self-consciousness, .66 for impulsiveness, and .81 for vulnerability.

\section{Statistical Analysis}

To test if dysfunctional beliefs and (poor) subjective sleep quality mediated the effects of the neuroticism (factor and traits) on insomnia, bivariate correlation and multiple regression analyses were conducted and path analysis models were tested with AMOS22. We first calculated bivariate correlations between sleep-related cognitions and insomnia, neuroticism (factor and traits) and insomnia, and neuroticism (factor and traits) and sleep-related cognitions. Given these correlations, we then conducted several regression and path analyses to test the mediating effects of sleep-related cognitions (as mediators) on the relationship between neuroticism (factor and traits) (as predictors) and insomnia (as DV). This dual approach was recommended by Holmbeck ${ }^{21}$ who adjusted Baron and Kenny's ${ }^{22}$ approach to statistical mediation analysis for path analysis and structural equation modeling (SEM). We used SEM in this study because: (1) in SEM models consisting of multiple predictors and/or mediators, all the relevant paths are directly and simultaneously tested (controlling for measurement error); (2) it provides several fit indices (eg, the chi-square statistic with degrees of freedom $\left(\chi^{2}\right.$ with $\left.\mathrm{df}\right)$, the comparative fit index (CFI), the root mean square error of approximation (RMSEA)) that inform about the goodness-of-fit of the mediational models and allow for comparing them (whether full or partial); (3) it incorporates a bootstrap framework that provides $p$-values and confidence intervals of the indirect or mediated effects that outperform the more classical Sobel tests, especially when sample sizes are moderate or small and when mediator and/or outcome variables are 
Table 2

Standardized Regression Weights ( $\beta$ ), Standard Errors (SE), p-values from Multiple Regression Analyses with Dysfunctional Beliefs (Second Column), Subjective Sleep Quality (Third Column), and Insomnia (Fourth Column) as Criteria and Neuroticism (Factor and Traits) as Predictors

\begin{tabular}{|c|c|c|c|c|c|c|c|c|c|c|}
\hline \multirow{2}{*}{\multicolumn{2}{|c|}{ Neuroticism }} & \multicolumn{3}{|c|}{ Dysfunctional Beliefs } & \multicolumn{3}{|c|}{ Subjective Sleep Quality } & \multicolumn{3}{|c|}{ Insomnia } \\
\hline & & $\beta$ & SE & $\mathbf{p}$ & $\beta$ & SE & $\mathbf{p}$ & $\beta$ & SE & $\mathbf{p}$ \\
\hline \multirow{7}{*}{$\begin{array}{l}\text { Factor } \\
\text { Traits }\end{array}$} & & .46 & .05 & $* * *$ & .38 & .05 & $* * *$ & .34 & .05 & $* * *$ \\
\hline & Anxiety & .18 & .07 & $*$ & .33 & .08 & $* * *$ & .21 & .08 & $* *$ \\
\hline & Angry-Hostility & .04 & .06 & .45 & .07 & .06 & .27 & .13 & .06 & $*$ \\
\hline & Depression & .17 & .09 & $*$ & .10 & .09 & .26 & .10 & .09 & .28 \\
\hline & Self-Consciousness & .02 & .07 & .73 & -.18 & .07 & $*$ & -.25 & .07 & $* * *$ \\
\hline & Impulsiveness & -.02 & .05 & .76 & -.04 & .05 & .49 & -.01 & .05 & .90 \\
\hline & Vulnerability & .19 & .07 & $* *$ & .17 & .07 & $*$ & .29 & .07 & $* * *$ \\
\hline
\end{tabular}

not normally distributed (which is the case for our dichotomous DV Insomnia). ${ }^{21,23}$

\section{RESULTS}

Associations between Sleep-Related

Cognitions and Insomnia; Associations among

Neuroticism (Factor and Traits) and Insomnia, Dysfunctional Beliefs, and Subjective Quality of Sleep

The dysfunctional beliefs and subjective sleep quality variables were positively associated with insomnia $\left(\mathrm{r}_{\mathrm{pb}}=.40, \mathrm{p}<.01\right.$ and $\mathrm{r}_{\mathrm{pb}}=.65, \mathrm{p}<.01$, respectively). Individuals reporting high levels of neuroticism were more likely to be insomniac, to report more dysfunctional beliefs and a poorer subjective quality of sleep than those with a low score of neuroticism. The 6 traits of neuroticism were positively correlated with insomnia, dysfunctional beliefs, and subjective sleep quality. Table 1 shows correlations between neuroticism and sleep-related variables.

Effects of Neuroticism (Factor and Traits) on Sleep-Related Variables: Insomnia,

Dysfunctional Beliefs, and Subjective Sleep Quality

Neuroticism: factor level. As Table 2 shows (factor level), the first set of regression analyses revealed statistically significant positive relations among neuroticism and dysfunctional beliefs, subjective sleep quality, and insomnia.

Next, when the neuroticism, dysfunctional beliefs and subjective sleep quality variables (as predictors) were entered simultaneously in a multiple regression analysis (with insomnia as DV), the effect of neuroticism on insomnia was reduced to nonsignificance (from $\beta=.34, p<.01$ in the regression model without any mediator to $\beta=.07, \mathrm{NS}$ in the regression model with mediators), whereas the ef- fects of dysfunctional beliefs and subjective sleep quality on insomnia were statistically significant $(\beta$ $=.14$ and $\beta=.56, \mathrm{p}<.01)$. According to Baron and Kenny $^{22}$ or Holmbeck, ${ }^{21}$ this set of findings constitutes evidence for perfect or full mediation: the effect of neuroticism on insomnia was fully mediated via dysfunctional beliefs and subjective sleep quality. Using these findings, we developed a first path model of insomnia. Figure 1 (top) illustrates this first model.

In this model, $43.8 \%$ of the variance of insomnia was explained. Fit indices indicated an excellent model fit: $\chi^{2}(\mathrm{df})=1.94(1)$; CFI $=.99$ (> .95 recommended cut-off); RMSEA $=.05$ (<.06 recommended cut-off) ${ }^{24}$ As deduced from the previous regression analyses, there was no more significant direct effect from the neuroticism factor to insomnia but 2 significant indirect effects through dysfunctional beliefs and (poor) subjective sleep quality. Table 3 (factor level) shows the standardized effects (total and indirect) and their significance, as well as the standardized confidence intervals of the indirect effects for the factor-level model.

In other words, because the direct effect of neuroticism on insomnia in the regression model without mediators disappeared in the path model, this pattern of results showed a significant combined full mediation through dysfunctional beliefs and (poor) subjective sleep quality. The comparison between this full-mediated path model and the partial-mediated path model (direct effect from neuroticism to insomnia not constrained to be zero) provided further evidence for full mediation: the difference between the amount of variance of insomnia explained by the partial-mediated model $\left(\mathrm{R}_{\text {partial }}^{2}=.442\right)$ and the one explained by the fullmediated model $\left(\mathrm{R}^{2}\right.$ full $\left.=.438\right)$ was not significant $\left(\mathrm{F}_{1,314}=2.28, \mathrm{NS}\right)$.

Neuroticism: trait level - anxiety, angry-hos- 
Figure 1

Path Models of Insomnia at the Factor Level (Top) and at the Trait Level (Down)
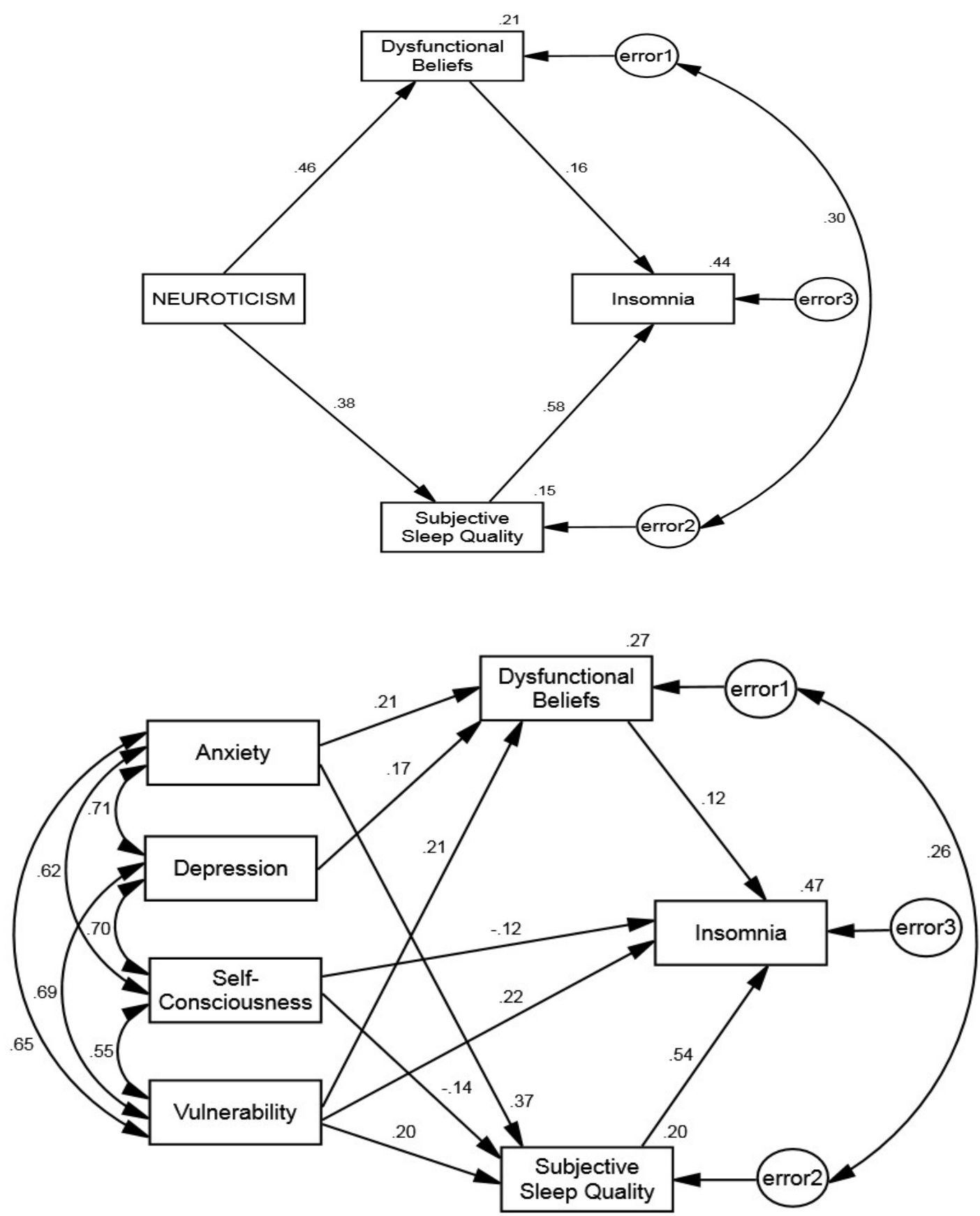

Note.

The Values above One-headed Arrows are Standardized Regression Coefficients. The Values at Double-headed Arrows are Correlations. Numbers above the Rectangles Reflect the Amount of Explained Variance of the Corresponding Variables. 


\begin{tabular}{|c|c|c|c|c|c|c|c|c|}
\hline \multicolumn{9}{|c|}{$\begin{array}{c}\text { Table } 3 \\
\text { Standardized Effects (Total and Indirect), Significance of the Standardized } \\
\text { Effects (Total and Indirect), Standardized Confidence Intervals of the Indirect } \\
\text { Effects (Factor Level and Trait Level) }\end{array}$} \\
\hline & \multicolumn{3}{|c|}{ Total Effects on } & \multicolumn{3}{|c|}{ Indirect Effects on Insomnia Via } & \multicolumn{2}{|c|}{$\begin{array}{c}\text { Confidence } \\
\text { Intervals }\end{array}$} \\
\hline & $\begin{array}{c}\text { Dysfunctional } \\
\text { Beliefs } \\
\end{array}$ & $\begin{array}{l}\text { Subjective } \\
\text { Sleep } \\
\text { Quality }\end{array}$ & Insomnia & $\begin{array}{c}\text { Dysfunctional } \\
\text { Beliefs }\end{array}$ & & $\begin{array}{l}\text { Subjective } \\
\text { Sleep } \\
\text { Quality } \\
\end{array}$ & Lower & Upper \\
\hline \multicolumn{9}{|l|}{ Factor Level } \\
\hline Neuroticism & $.46^{* *}$ & $.38 * *$ & $.29 * *$ & & $.29 * *$ & & .23 & .37 \\
\hline \multicolumn{9}{|l|}{ Trait Level } \\
\hline Anxiety & $.21^{*}$ & $.37 * *$ & $.23 * *$ & & $.23 * *$ & & .14 & .32 \\
\hline Depression & $.17^{*}$ & - & $.02 *$ & $.02 *$ & & - & .01 & .06 \\
\hline Self-Consciousness & - & $-.14 *$ & $-.20 * *$ & - & & $-.08^{*}$ & -.15 & -.01 \\
\hline Vulnerability & $.21 * *$ & $.20 * *$ & $.35^{* *}$ & & $.13 * * *$ & & .06 & .21 \\
\hline \multicolumn{9}{|c|}{$* \mathrm{p}<.05 ; * * \mathrm{p}<.01 ; * * * \mathrm{p}<.001$} \\
\hline
\end{tabular}

tility, depression, self-consciousness, impulsiveness, and vulnerability. As shown in Table 2 (trait level), several traits of neuroticism were associated with dysfunctional beliefs, subjective sleep quality, or/and insomnia. More precisely, statistically significant effects in the directions predicted (except for the self-consciousness trait) were observed among the anxiety, depression, vulnerability traits and dysfunctional beliefs, among the anxiety, self-consciousness, vulnerability traits and subjective sleep quality, as well as among the anxiety, angry-hostility, self-consciousness, vulnerability traits and insomnia (for the self-consciousness trait, regression analyses revealed negative effects on both subjective sleep quality and insomnia whereas correlation analyses showed positive associations with these variables. These reversed signs suggest a negative suppression that could reflect a statistical artifact probably due to the low correlation between self-consciousness and insomnia $\left[\mathrm{r}_{\mathrm{pb}}=.16\right]$ combined with a strong mean correlation between this trait and the 5 other traits of neuroticism $\left.\left[\mathrm{r}_{\text {mean }}=.50\right]\right)$.

Next, insomnia was regressed on both the neuroticism traits and the mediators - dysfunctional beliefs and subjective sleep quality. Results from this analysis showed: (1) significant effects of dysfunctional beliefs $(\beta=.11, \mathrm{p}<.05)$ and subjective sleep quality $(\beta=.52, \mathrm{p}<.001)$ on insomnia; $(2 \mathrm{a})$ reduced but still significant effects of self-consciousness (from $\beta=-.25, \mathrm{p}<.001$ in the regression model without any mediator to $\beta=-.16, \mathrm{p}<.01$ in the regression model with mediators) and vulnerability (from $\beta=.29, p<.001$ to $\beta=.18, p<.01$ ) on insomnia; (2b) no more significant effect of anxiety (from $\beta=.21, \mathrm{p}<.01$ to $\beta=.01, \mathrm{NS}$ ) and angry-hostility (from $\beta=.13, p<.05$ to $\beta=.09, \mathrm{NS}$ ) on insomnia. In this way, the conditions imposed by Baron and Kenny ${ }^{22}$ or Holmbeck ${ }^{21}$ to establish mediation were fulfilled. That is, dysfunctional beliefs and/ or subjective sleep quality fully mediated the relation between anxiety and insomnia, and partially mediated the self-consciousness-insomnia and vulnerability-insomnia paths. The direct effect of angry-hostility on insomnia in the regression model without mediators disappeared when we added dysfunctional beliefs and subjective sleep quality; however, there was no mediation as we observed no significant effect of angry-hostility on these 2 variables. In the same vein, depression had "only" an indirect effect on insomnia through dysfunctional beliefs, but it was not a mediated effect as there was no significant direct effect from depression to insomnia in the regression model without mediators. Based on these results, we developed a second path model of insomnia. Figure 1 (down) illustrates this second model. In this model, $46.8 \%$ of the variance of insomnia was explained. Fit indices indicated an excellent fit of this model $\left(\chi^{2}(\mathrm{df})=\right.$ $3.12(4) ; \mathrm{CFI}=1.00 ; \mathrm{RMSEA}=.00)$. As suggested by the previous regression analyses, this model was essentially a partial-mediated path model because 2 significant direct effects remained between the self-consciousness and vulnerability traits and insomnia. Table 3 (trait level) shows the standardized effects (total and indirect) and their significance, as well as the standardized confidence intervals of the indirect effects for this trait-level model. The 
comparison between this partial-mediated path model and the full-mediated path model (direct effect from these 2 traits to insomnia constrained to be zero) provided further evidence for partial mediation. That is, the chi-square statistic of the partial-mediated model was significantly lower than the chi-square statistic of the full-mediated model $\left(\Delta \chi^{2}(2)=16.98, \mathrm{p}<.001\right)$.

\section{DISCUSSION}

The present research aimed to explore predisposing and perpetuating mechanisms of insomnia. As such, the relationship among neuroticism (and then neuroticism-related traits), sleep-related cognitions, and insomnia has been investigated through mediation analyses, to answer 2 questions: (1) Does neuroticism contribute to insomnia directly or through sleep-related cognitions? (2) Is the mechanism of the relationship between neuroticism and insomnia different depending on neuroticism-related traits or the same? Our ultimate goal was to propose structured path models in which dysfunctional beliefs and poor subjective sleep quality mediate the relationship between neuroticism (factor and traits) and insomnia. A better understanding of these questions could lead to enhanced efficiency of therapeutic care in CBT-I.

To address these issues, we used the NEO-PI$\mathrm{R}$, a questionnaire measuring 5 genetically-linked personality factors. We restricted our research to one domain - Neuroticism - because it has been reported to be the most associated with insomnia. ${ }^{5}$ The choice of this personality inventory constitutes an original perspective because: (1) few studies have used it in this context; $;^{5}(2)$ it refers to the trait theory that conceives personality as a genetic predisposition for a large part; ${ }^{25}$ and (3) it represents the first attempt to document the relationship between neuroticism and insomnia not only at the factor level but also at the trait level.

In a preliminary step, we conducted several correlation analyses that confirmed our general hypotheses. As expected, statistically significant positive associations were found between the 2 sleep-related cognitions and insomnia. The more an individual reported dysfunctional beliefs and poor subjective sleep quality, the more he/she suffered insomnia. These results concur with previous studies that observed similar relations. ${ }^{26,27}$ Correlation analyses also revealed a clear pattern of positive relationship among neuroticism (at the factor and trait levels) and insomnia, dysfunctional beliefs, and subjective sleep quality. That is, the more an individual displayed neurotic traits associated with anxiety, angry-hostility, depression, self-consciousness, impulsiveness, and vulnerability, the more he/she experienced insomnia associated with dysfunctional beliefs and poor subjective sleep quality. Leblanc et $\mathrm{al}^{6}$ found similar results.

Accordingly, these results allowed us to implement the dual approach for testing mediated effects recommended by Holmbeck. ${ }^{21}$ At the factor level, neuroticism showed a significant positive direct effect on insomnia as well as on dysfunctional beliefs and subjective sleep quality. After controlling for these 2 sleep-related cognitions, the impact of neuroticism on insomnia disappeared, whereas the effects of dysfunctional beliefs and subjective sleep quality on insomnia were significant, allowing us to answer the first research question - dysfunctional beliefs and subjective sleep quality work as mediators of the relation between neuroticism and insomnia. The goodness-of-fit of the full-mediated path model deduced from these findings, as well as the significance of the indirect effects on insomnia, confirmed the combined full mediation of the effect of neuroticism on insomnia through dysfunctional beliefs and poor subjective sleep quality. Therefore, at the factor level, our mediational hypothesis was supported by these data. This result is in line with: (1) Morin's ${ }^{28}$ model that describes the relationship between dysfunctional cognitions (worry about sleep loss) and insomnia.11 (2) Calkins et al's ${ }^{14}$ findings that demonstrate an additive contribution of neuroticism and dysfunctional beliefs in predicting sleep disturbance, (3) Harvey et al's ${ }^{27}$ approach that emphasizes the role of distorted perception of sleep in the development of insomnia. More importantly, our result represents an important contribution to the comprehension of insomnia, because it combines and prioritizes neuroticism and sleeprelated cognitions within only one mediational model. A similar result has been recently reported by Gurtman et al, ${ }^{15}$ demonstrating the mediating role of dysfunctional beliefs in the relationship between neuroticism and insomnia.

At the trait level, 5 of the 6 traits of neuroticism (all but impulsiveness) were found to be significant predictors of one, 2 , or 3 sleep-related variables: the anxiety and vulnerability traits had a consistent direct effect on insomnia, dysfunctional beliefs, and subjective sleep quality; we also observed a direct effect of self-consciousness on insomnia and subjective sleep quality, a direct effect of depression on dysfunctional beliefs, and a direct effect of angry-hostility on insomnia. Regressing insomnia on both the traits of neuroticism and the 2 sleep-related cognitions revealed different patterns of relationship depending on the neuroticism-related traits. That is, dysfunctional beliefs and/or subjective sleep quality fully mediated the anxiety-insomnia path, and partially mediated the vulnerability-insomnia and self-consciousnessinsomnia paths; by contrast, we only observed an indirect, non-mediating effect of depression on insomnia through dysfunctional beliefs, whereas the direct effect of angry-hostility on insomnia no longer reached statistical significance. Here again, the goodness-of-fit of the partial-mediated path model deduced from these findings, as well as the significance of the indirect effects on insomnia, confirm this set of findings. Therefore, at the trait level, our mediational hypothesis may be considered as partly supported by these data. 
Most of our data are in congruence with previous studies. With regard to anxiety and depression, our results agree with Leblanc et $\mathrm{al}^{6}$ who found that people characterized by higher depressive and anxiety symptoms were at greater risk of developing insomnia than normal sleepers; in addition, the full-mediated effect of anxiety on insomnia notably through subjective sleep quality reinforces the results obtained by Tang and Harvey, ${ }^{29}$ suggesting that anxiety may be considered as a determinant of misperception of sleep (see also Harvey et $\mathrm{al}^{27}$ ). The direct and mediated effect of vulnerability on insomnia supports the role played by the appraisal of stressors and the perceived lack of control over stressful events on insomnia as suggested by Morin et al. ${ }^{10}$ For the angry-hostility trait, its direct effect on insomnia confirms previous research, ${ }^{8}$ even though, in our conditions, it was reduced to non-significance in the presence of the 2 mediators. Indeed, given the strength of the $\mathrm{p}$-value of the regression coefficient $(\mathrm{p}=.07$, close to significance), we speculate that this non-significance was mainly due to our sample characteristics. Further investigations using an independent sample are needed to test this hypothesis. By contrast, and unlike Wang et al, ${ }^{9}$ we could not find any effect of impulsiveness on insomnia. This discrepancy could be due to the use of different measures and different statistical techniques. Our result makes sense to us given that, in the bivariate correlations, the associations between impulsiveness and the 3 sleep-related variables were the lowest ones. This could be explained by the relatively low reliability of this trait in our sample $(\alpha=.66<.70$ cut-off value). Finally, we found a positive correlation between self-consciousness and insomnia, as well as direct and indirect effects of this trait on insomnia. At first glance, this finding provides new evidence supporting the "internalization" hypothesis suggesting that insomniacs tend to internalize their feelings and repress their conflicts and stress. ${ }^{5,11}$ However, the negative sign of the (direct and indirect) effects of self-consciousness on insomnia tends to undermine this interpretation somewhat, unless this reverse sign is an artifact due to our sample characteristics. Future studies with a larger sample should help to clarify this important point. Once again, the set of organized relationships among neuroticism-related traits, sleep-related cognitions, and insomnia constitutes a major finding and an original contribution of this study - we highlight a structured path model of these psychological factors and their impact on the emergence of insomnia.

The consequences for therapeutic strategies are important, because our results suggest some tracks to guide the cognitive component of CBT-I. In the context of insomnia, cognitive therapy seeks to change sleep expectations, perceived causes and consequences of insomnia, and beliefs about sleeppromoting practices. ${ }^{30}$ We showed that the impact of neuroticism on insomnia is mediated primarily by dysfunctional beliefs and subjective sleep quality. Anxiety and vulnerability, and to lesser degree, depression, are the most important traits involved in this mediational process. Therefore, the focus of the cognitive therapy should first address cognitions linked with anxiety related to misperception of sleep, which is the most important determinant likely to produce and maintain insomnia. Vulnerability also should be addressed quickly in the cognitive approach of CBT-I. This could be fulfilled by implementing some aspects of Acceptation and Commitment Therapy ${ }^{31}$ as well as Mindfulness Based Stress Reduction, ${ }^{32}$ new approaches more likely to help patients with dysfunctional cognitions related to appraisal of stressors and lack of control. Our results do not rule out the classical approach of cognitive therapy. However, it does possible new priorities in the determinants of insomnia.

This study has some limitations. First, as previously mentioned, further (cross-validated) studies are needed to clarify the strength of the impact of angry-hostility on insomnia and the sign of the selfconsciousness-insomnia path. Second, the reliance on cross-sectional self-report measures is a limitation, particularly in defining insomnia. Objective measures of insomnia, such as polysomnography or at least actigraphy, would be useful to confirm the present data even though the present tools have been validated and are widely used in the field of insomnia. ${ }^{11}$ In the same way, it is not possible to exclude that some patients experienced depression as a comorbid disorder associated to insomnia as sleep debt by itself produces depressive symptoms. We meet here the difficulty of defining primary insomnia because insomnia is almost always present in depression as a pathognomonic symptom. We speculate however, that for most participants of the insomnia group, this was not the case because they assessed themselves as non-depressive. Third, in spite of the use of the NEO-PI-R, a measure of personality traits considered as genetic predispositions, and the mediational status of the relationship between neuroticism and insomnia as shown here, some different or complementary paths relating insomnia to other variables (eg, reciprocal effects or feedback loops) cannot be excluded, because our results are derived from nonexperimental data. ${ }^{23}$ Therefore, as Van de Laar et $\mathrm{al}^{5}$ suggest, longitudinal studies are needed. Fourth, although this would exceed the scope of the present study, a closer look at perfectionism and extraversion as other personality traits would be fruitful in exploring the relationship between personality and insomnia through mediators, ${ }^{33}$ or even completing our mediational model. Finally, although the sample size was adequate for performing our analyses, future studies could be more extensive by including larger samples. This would have the advantage of allowing for additional investigations, such as those previously mentioned, and enhancing the generalizability of our findings.

Nevertheless, this research contributes to clarify- 
ing the role of neuroticism in the onset of primary insomnia. In identifying the relationship among neuroticism (both at the factor and trait levels), dysfunctional beliefs, subjective sleep quality, and insomnia through fully- or partially-mediated path models, we offer some insight about the way a number of vulnerability factors for insomnia are organized and impact each other. As previously specified, this knowledge could be helpful in configuring prevention and intervention programs to treat insomnia, especially in its cognitive component.

\section{Human Subjects Approval Statement}

The study protocol was approved by the University of Strasbourg Institutional Review Board.

\section{Conflict of Interest Disclosure Statement}

The authors declare that they have no conflicts of interest.

\section{Acknowledgments}

The authors thank all respondents for their valuable participation.

\section{References}

1. American Psychiatric Association (APA). Diagnostic and Statistical Manual of Mental Disorders DSM-IV-TR. 4th ed. Washington, DC: APA; 2000.

2.Duggan KA, Friedman HS, McDevitt EA, Mednick SC Personality and healthy sleep: the importance of conscientiousness and neuroticism. PLOS One. 2014;9(3):1-11.

3. Gau SF. Neuroticism and sleep-related problems in adolescence. Sleep. 2000;23(4):495-502.

4. Harvey CJ, Gehrman P, Espie CA. Who is predisposed to insomnia: a review of familial aggregation, stressreactivity, personality and coping style. Sleep Med Rev. 2014;18(3):237-247.

5. Van de Laar M, Verbeek I, Pevernagie D, et al. The role of personality traits in insomnia. Sleep Med Rev. 2010;14(1):61-68

6. LeBlanc M, Mérette C, Savard J, et al. Incidence and risk factors of insomnia in a population-based sample. Sleep. 2009;32(8):1027-1037.

7. Nguyen-Rodriguez ST, Lisha NE, Spruijt-Metz D, et al. Coping mediates the effects of depressive symptoms on sleep problems. Am J Health Behav. 2015;39(2):183-190.

8. Ottoni GL, Lorenzi TM, Lara DR. Association of temperament with subjective sleep patterns. J Affect Disord. 2011;128(1-2):120-127.

9. Wang W, Zhu SZ, Pan LC, et al. Mismatch negativity and personality traits in chronic primary insomniacs. Funct Neurol. 2001;16(1):3-10.

10. Morin CM, Rodrigue S, Ivers H. Role of stress, arousal, and coping skills in primary insomnia. Psychosom Med. 2003;65(2):259-267.

11. Park JH, An H, Jang ES, Chung S. The influence of personality and dysfunctional sleep-related cognitions on the severity of insomnia. Psychiatry Res. 2012;197(3):275279.

12. Yan $Y$, Lin $R$, Tang $X$, et al. The relationship between worry tendency and sleep quality in Chinese adolescents and young adults: the mediating role of state-trait anxiety. J Health Psychol. 2014;19(6):778-788

13. Harvey AG. A cognitive model of insomnia. Behav Res
Ther. 2002:40(8):869-893

14. Calkins AW, Hearon BA, Capozzoli MC, et al. Psychosocial predictors of sleep dysfunction: the role of anxiety sensitivity, dysfunctional beliefs, and neuroticism. Be hav Sleep Med. 2013;11(2):133-143.

15. Gurtman CG, McNicol R, McGillivray JA. The role of neuroticism in insomnia. Clinical Psychologist. 2014;18:116124

16. Fernandez-Mendoza J, Calhoun S, Bixler EO, et al. Sleep misperception and chronic insomnia in the general population: role of objective sleep duration and psychological profiles. Psychosom Med. 2011;73(1):88-97.

17. Morin CM. Dysfunctional beliefs and attitudes about sleep: preliminary scale development and description. The Behavior Therapist. 1994;17:163-164.

18. Buysse DJ, Reynolds CF, Monk TH, et al. The Pittsburgh Sleep Quality Index: a new instrument for psychiatric practice and research. Psychiatry Res. 1989;28(2):193213.

19. Costa PT, McCrae RR. Revised NEO Personality Inventory (NEO-PI-R) and NEO Five-Factor Inventory (NEO-FFI): Professional Manual. Lutz, FL: Psychological Assessment Resources; 1992.

20. McCrae RR, Costa PT. A contemplated revision of the NEO Five-Factor Inventory. Pers Individ Diff. 2004;36(3):587596.

21. Holmbeck GN. Toward terminological, conceptual, and statistical clarity in the study of mediators and moderators: examples from the child-clinical and pediatric psychology literatures. J Consult Clin Psychol. 1997;65(4):599-610.

22. Baron RM, Kenny DA. The moderator-mediator variable distinction in social psychology research: conceptual, strategic, and statistical considerations. J Pers Soc Psychol. 1986;51(6):1173-1182.

23. Shrout PE, Bolger N. Mediation in experimental and nonexperimental studies: new procedures and recommendations. Psychol Methods. 2002;7(4):422-445.

24. Hu L, Bentler PM. Cut-off criteria for fit indexes in covariance structure analysis: conventional criteria versus new alternatives. Structural Equation Modeling. $1999 ; 6(1): 1-55$.

25. McCrae RR, Costa PT. Empirical and theoretical status of the Five-Factor Model of personality traits. In Boyle GJ, Matthews G, Saklofske DH, eds. SAGE Handbook of Personality Theory and Assessment. Thousand Oaks, CA: SAGE Publications 2008:273-294.

26. Epsie CA. Insomnia: Conceptual issues in the development, persistence, and treatment of sleep disorder in adults. Annu Rev Psychol. 2002;53:215-243.

27. Harvey AG, Tang N, Browning L. Cognitive approaches to insomnia. Clin Psychol Rev. 2005;25(5):593-611.

28. Morin CM. Insomnia: Psychological Assessment and Management. New York, NY: Guilford Press; 1993.

29. Tang N, Harvey AG. Effects of cognitive arousal and physiological arousal on sleep perception. Sleep. 2004;27(1):69-78.

30. Morin CM, Espie CA. Insomnia. A Clinical Guide to Assessment and Treatment. New York, NY: Springer; 2004.

31. Hayes CC. Acceptation and Commitment Therapy, Relational Frame Theory, and the third wave of behavior therapy. Behav Ther. 2004;35:639-665.

32. Kabat-Zinn J. Full Catastrophe Living: Using the Wisdom of the Body and the Mind to Face Stress, Pain and Ilness. New York, NY: Delacorte Press; 1990.

33. Azevedo MH, Bos SC, Soares MJ, et al. Longitudinal study on perfectionism and sleep disturbance. World $J$ Biol Psychiatry. 2010;11(2):476-485. 